\title{
Effect of Reaction Conditions on Production of Catechinone Hair Dyestuff in Water/Alcohol Mixed Solution
}

\author{
Takanori Matsubara1, Isao Wataoka², Hiroshi Urakawa², Hidekazu Yasunaga ${ }^{2 *}$ \\ ${ }^{1}$ Department of Mechanical Engineering, College of Industrial Technology, Amagasaki, Japan \\ ${ }^{2}$ Department of Biobased Materials Science, Kyoto Institute of Technology, Kyoto, Japan \\ Email: yasunaga@kit.ac.jp
}

Received 28 May 2014; revised 20 June 2014; accepted 27 June 2014

Copyright (C) 2014 by authors and Scientific Research Publishing Inc.

This work is licensed under the Creative Commons Attribution International License (CC BY). http://creativecommons.org/licenses/by/4.0/

(c) (i) Open Access

\begin{abstract}
Catechinone hair dyestuff was produced chemically from (+)-catechin by the oxidation with $\mathrm{O}_{2}$ in basic water/alcohol mixed solution. The effects of reaction conditions such as basicity, sorts of added base, temperature, alcohol molar fraction of solvent and sorts of added alcohols on the catechinone formation were studied in order to increase the efficiency of the dyestuff production. The amount of obtained catechinone increases with increasing basicity of the reaction solution. The dyestuff is obtained by adding monoethanol amine, diethanol amine, triethanol amine, L-arginine, $\mathrm{Na}_{2} \mathrm{CO}_{3}, \mathrm{~K}_{2} \mathrm{CO}_{3}, \mathrm{Na}_{3} \mathrm{PO}_{4}$ or $\mathrm{NaOH}$ into the solution as a base and the most preferable base is monoethanol amine. The optimum temperature for the production in water/ethanol solution is $30^{\circ} \mathrm{C}$. It was found that water-soluble alcohols such as methanol, ethanol, 2-propanol, 1-propanol and tert-butyl alcohol are available for preparing the dyestuff. The optimum alcohol molar fraction of the mixed solvent used for the dye formation is 0.45 for methanol, 0.25 for ethanol and 0.20 for 2-propanol, 0.15 for 1-propanol and 0.10 for tert-butyl alcohol. The amount of the obtained dyestuff reaches a maximum at 1) 0.35 and $0.59,2) 0.35$ or 3) $0.35 \mathrm{~mol} \cdot \mathrm{kg}^{-1}$ of the concentration of (+)-catechin for the 1) water/methanol, 2) water/ethanol or 3) water/1-propanol system, respectively.
\end{abstract}

\section{Keywords}

Catechinone, Hair Dyestuff, (+)-Catechin, Chemical Preparation, Water/Alcohol Mixed Solution

\section{Introduction}

A great number of people dye their hair in the world today. Above all, human hair dyeing by using oxidation

\footnotetext{
*Corresponding author.
}

How to cite this paper: Matsubara, T., Wataoka, I., Urakawa, H. and Yasunaga, H. (2014) Effect of Reaction Conditions on Production of Catechinone Hair Dyestuff in Water/Alcohol Mixed Solution. Advances in Chemical Engineering and Science, 4, 292-299. http://dx.doi.org/10.4236/aces.2014.43032 
dyes is most frequently employed. The advantages of the permanent hair colouring technique using the oxidation dye are the higher dyeability for darker hair and higher colour fastness to washing [1] [2]. However, many kinds of symptoms are caused for some people after using of the oxidation hair dyes (For example, [3] [4]). The problems should be solved for better human life also by scientific way.

Under such the situation, the authors invented catechinone (4-(3,4-dihydro-3 $\alpha, 5,7-$ trihydroxy-2H-1-benzopyran-2 $\alpha$-yl) 1,2-benzoquinone) as a safer hair dyestuff and it is prepared by oxidising (+)-catechin [5]. The obtained colourant from $(+)$-catechin contains a small amount of byproducts but the main product is catechinone, of which structure is shown in Figure 1. Catechinone dyes human hair orange, reddish orange and deep yellowish brown. The colour fastness of the dyed hair to washing and light is high enough for practical use. Catechinone does not cause erythema or oedema on skin of rabbits. The dyestuff is obtained by enzymatic [5] and chemical [6] oxidation methods. It is produced by using a catechol oxidase such as tyrosinase and ascorbic acid oxidase, and the dye formation rate and the reaction selectivity of the enzymatic reactions are high. On the other hand, it is prepared by using $\mathrm{O}_{2}$ gas in basic solution, where the formation rate is lower than that of the enzymatic method. However, the chemical method is easier to control and useful industrially because of the low cost. The catechinone formation occurs via proton dissociations and one electron oxidations by oxygen, thus the production efficiency of the dyestuff is improved by increasing $\mathrm{pH}$ of aqueous solution and the concentration of $\mathrm{O}_{2}$ [6].

Nevertheless, the yield of catechinone is not high in one batch of the reaction solution because of the low solubility of $(+)$-catechin in an aqueous solution, and then it should be improved for practical use. The solubility of $(+)$-catechin in ethanol is over 30 times higher than that in water. Then, the chemical dyestuff preparation was attempted by adding ethanol into the reaction solution and increasing the concentration of $(+)$-catechin to increase the dye production efficiency in the previous study [7]. The results showed that catechinone is not formed in pure ethanol solution but it is obtained in the water/ethanol mixed solution at 0.28 of the ethanol molar fraction of the solvent. The amount of the dyestuff formed in water/ethanol mixed solution is over 20 times larger than that in the aqueous solution system. It was suggested that water-ethanol mixing ratio of the solvent is an important factor for the formation efficiency. The other reaction conditions such as basicity and temperature are considered to be the key factor for the formation, too. It is also interesting to investigate the availability of other water-soluble alcohols for the reaction and the effect of the alkyl group structure of the alcohol on it.

In this study, the relationships between the amount of the formed catechinone and the dye preparation conditions such as basicity, sorts of added base, temperature, alcohol molar fraction of solvent and sorts of added alcohols were studied for the oxidation reaction of (+)-catechin in water/alcohol solution.

\section{Experimental}

\subsection{Materials}

$(+)$-Catechin and other reagents described below were purchased from Sigma and Nacalai tesque, respectively. (+)-Catechin hydrate, ethanol (EtOH, 99.5\%), methanol (MeOH, 99.8\%), 1-propanol (1-PrOH, 99.5\%), 2-propanol (2-PrOH, 99.7\%) and tert-butyl alcohol $(t-\mathrm{BuOH}, 99.0 \%)$ were used without further purification. Monoethanol amine (MEA), diethanol amine (DEA), triethanol amine (TEA), L-arginine (Arg), sodium carbonate $\left(\mathrm{Na}_{2} \mathrm{CO}_{3}\right)$, potassium carbonate $\left(\mathrm{K}_{2} \mathrm{CO}_{3}\right)$, sodium phosphate $\left(\mathrm{Na}_{3} \mathrm{PO}_{4}\right)$ and sodium hydroxide $(\mathrm{NaOH})$ were used as base without further purification.<smiles>O=C1C=CC(C2Oc3cc(O)cc(O)c3CC2O)=CC1=O</smiles>

Figure 1. Chemical structure of catechinone. 


\subsection{Dyestuff Preparation}

$(+)$-Catechin and a base were dissolved in $100 \mathrm{~g}$ of distilled water/alcohol mixed solvent. Oxygen gas $(\geq 99.5$ vol\%) was introduced continuously into the reaction solution through porous glass ball filter (pore size: 40 - 50 $\mu \mathrm{m})$ at $100 \mathrm{ml} \cdot \mathrm{min}^{-1}$ of flow rate, and the solution was stirred for prescribed time at $20^{\circ} \mathrm{C}-50^{\circ} \mathrm{C}$.

\subsection{Measurements}

The reaction solution was sampled at several time intervals and diluted by distilled water with a dilution ratio ( $f$ ). The ultraviolet-visible absorption spectra of the sampled solution were measured by a Hitachi U-3900H spectrophotometer at $25^{\circ} \mathrm{C}$. The dye formation behaviour was monitored by using the absorbance at $430 \mathrm{~nm}\left(A_{430}\right)$ at each reaction time $(t)$. The obtained $f \cdot A_{430}$ values correspond to the concentration of the formed dye [7] and were used the index of the amount of the dyestuff formed. The dyestuff formation rate $(v)$ was calculated by $v=$ $\left[\Delta\left(f \cdot A_{430}\right)\right]_{\max } / \Delta t$, where $\Delta t$ is the reaction time interval and $\left[\Delta\left(f \cdot A_{430}\right)\right]_{\max }$ the maximum increment of $f \cdot A_{430}$ for the $\Delta t$. Constant $\Delta t$ was adopted for the calculation of $v$ for each the experiment.

The apparent basicity of the reaction mixed solution $\left(\mathrm{pH}^{*}\right)$ was determined through the measurement by a TOA-DKK MM-60R multi water quality meter with a TOA-DKK ELP-031 glass combination electrode at $25^{\circ} \mathrm{C}$.

\section{Results and Discussion}

\subsection{Effects of Basicity and Sorts of Bases}

The colour of reaction solution turns orange and reddish brown with reaction time showing the formation of catechinone dyestuff. Catechinone is hardly obtained in acidic and neutral reaction solution without base. The rate of catechinone formation increases with increasing the basicity of aqueous reaction solution [6] and it is increased by using a stronger organic base [7]. The previous results indicate the strong influence of basicity on the reaction. Then, the effects of basicity and sort of bases on the catechinone formation were studied first. The $\mathrm{pH}$ is not defined in the water/organic solvent mixture, of which organic composition is high, and the apparent basicity was estimated as using $\mathrm{pH}^{*}$ in the study. The amount of the formed dyestuff at 200 min of reaction time $\left(\left(f \cdot A_{430}\right)_{200 \min }\right)$ and the dyestuff formation rate $(v)$ value in the water/ethanol mixed solution for each of the experiment with a variety of bases changing their concentration (molarity: $m_{\mathrm{B}}$ ) were measured and determined. The alcohol molar fraction of the mixed solvent $\left(x_{\mathrm{A}}\right)$ was 0.28 .

Figure 2 shows the relationship between $\mathrm{pH}^{*}$ and $\left(f \cdot A_{430}\right)_{200 \mathrm{~min}}$ for the water/ethanol solution with MEA. The resulting values are also summarised in Table 1 . The $\left(f \cdot A_{430}\right)_{200 \min }$ and $v$ increase with increasing $\mathrm{pH}^{*}$ and $m_{\mathrm{B}}$ of MEA as shown in the figure and for experimental number $2-5$ in the table. The results show that the reaction is

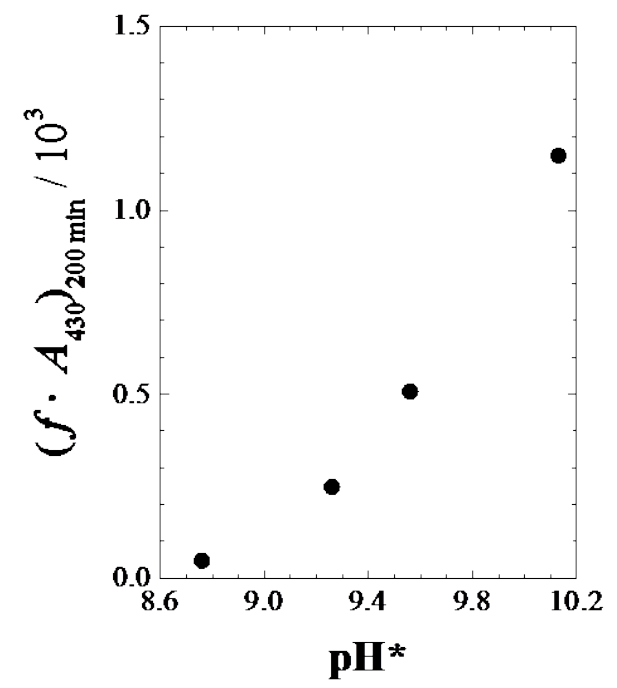

Figure 2. The $\mathrm{pH}^{*}$ dependence of the $\left(f \cdot A_{430}\right)_{200 \min }$ for the catechinone produced in water/ethanol solution $\left(x_{\mathrm{A}}\right.$ $=0.28$ ) with MEA at $30^{\circ} \mathrm{C}$. 
Table 1. The reaction condition and the resulting rate and amount of catechinone dye. The reactions are started in water/ethanol solution $\left(x_{\mathrm{A}}=0.28\right)$ at $m_{\mathrm{S}}=0.172 \mathrm{~mol} \cdot \mathrm{kg}^{-1}$ at $30^{\circ} \mathrm{C}$.

\begin{tabular}{ccccccc}
\hline & Base & $\mathrm{pK}_{\mathrm{b}}^{\mathrm{b}}$ & $m_{\mathrm{B}} / \mathrm{mol}^{\mathrm{b}} \mathrm{kg}^{-1}$ & $\mathrm{pH}^{* \mathrm{c}}$ & $v / \mathrm{s}^{-1}$ & $\left(f \cdot A_{430}\right)_{200 \mathrm{~min}}$ \\
\hline 1 & None & - & - & 4.84 & $\sim 0$ & 2.50 \\
2 & MEA & 4.49 & 0.010 & 8.76 & $6.23 \times 10^{-3}$ & 47.5 \\
3 & MEA & 4.49 & 0.050 & 9.26 & $2.76 \times 10^{-2}$ & 248 \\
$4^{\mathrm{a}}$ & MEA & 4.49 & 0.10 & 9.56 & $6.97 \times 10^{-2}$ & 507 \\
5 & $\mathrm{MEA}$ & 4.49 & 0.50 & 10.13 & $1.35 \times 10^{-1}$ & 1148 \\
$6^{\mathrm{a}}$ & $\mathrm{DEA}$ & 5.12 & 0.10 & 9.21 & $1.79 \times 10^{-2}$ & 133 \\
$7^{\mathrm{a}}$ & $\mathrm{TEA}$ & 6.24 & 0.10 & 8.65 & $2.72 \times 10^{-3}$ & 29.0 \\
8 & $\mathrm{Arg}$ & 4.91 & 0.10 & 9.34 & $2.54 \times 10^{-2}$ & $92.3^{\mathrm{d}}$ \\
9 & $\mathrm{Na}_{2} \mathrm{CO}_{3}$ & 3.68 & 0.10 & 9.76 & $1.41 \times 10^{-1}$ & 660 \\
10 & $\mathrm{~K}_{2} \mathrm{CO}_{3}$ & 3.68 & 0.10 & 10.15 & $1.62 \times 10^{-1}$ & 670 \\
11 & $\mathrm{Na}_{3} \mathrm{PO}_{4}$ & 1.68 & 0.0068 & 8.87 & $2.91 \times 10^{-3}$ & 23.5 \\
12 & $\mathrm{NaOH}^{2}$ & - & 0.10 & 10.06 & $1.70 \times 10^{-1}$ & 546
\end{tabular}

${ }^{\mathrm{a}}$ Previous work [7]; ${ }^{\mathrm{b}}$ The base dissociation constant in water at $25^{\circ} \mathrm{C}$ [8]; ${ }^{\mathrm{c}} \mathrm{Apparent} \mathrm{pH}$ value in the water/ethanol mixed solvent; ${ }^{\mathrm{d}} f \cdot A_{430}$ at $80 \mathrm{~min}$.

promoted by higher basicity of the reaction solution. This relationship is similar to that between $\mathrm{pH}$ and the rate of catechinone formation in aqueous solution [6]. The MEA is also more effective for the formation than DEA and TEA. The deprotonated (+)-catechin in a basic medium works as an electron donor and reacts with $\mathrm{O}_{2}$ easily [6] [9]. Therefore, the higher basicity should contribute to promote the formation reaction also in the water/ ethanol solution.

Furthermore, the preparation of the dyestuff was made in the mixed solution with basic amino acid L-arginine or inorganic bases such as $\mathrm{Na}_{2} \mathrm{CO}_{3}, \mathrm{~K}_{2} \mathrm{CO}_{3}, \mathrm{Na}_{3} \mathrm{PO}_{4}$ or $\mathrm{NaOH}$ (as listed in Table 1). The solubility of $\mathrm{Na}_{3} \mathrm{PO}_{4}$ in the water/ethanol mixed solution is low and its saturated concentration is $0.0068 \mathrm{~mol} \cdot \mathrm{kg}^{-1}$. The $m_{\mathrm{B}}$ of other inorganic bases was $0.10 \mathrm{~mol} \cdot \mathrm{kg}^{-1}$. The results in Table 1 show that $f \cdot A_{430}$ for the reaction system with $\mathrm{Na}_{2} \mathrm{CO}_{3}$, $\mathrm{K}_{2} \mathrm{CO}_{3}$ or $\mathrm{NaOH}$ is larger than that with same concentration of MEA. However, it is lower than that with 0.50 $\mathrm{mol} \cdot \mathrm{kg}^{-1}$ of MEA, and the solubility of inorganic bases in the water/ethanol mixed solution is lower as compared with that of MEA. The solution $\mathrm{pH}^{*}$ decreases sharply at the earlier stage of the reaction for the inorganic base systems. This results in the decrease of the reactivity. In contrast, the $\mathrm{pH}^{*}$ decreases gradually in the MEA system. Consequently, using MEA as the base is of greater advantage for the production.

\subsection{Effects of Temperature}

The rate of oxidation reactions are generally influenced by temperature. It is instructive to study the effect of reaction temperature on catechinone formation for obtaining better condition to produce the dyestuff efficiently. Figure 3 indicates the relationship between the reaction temperature $(T)$ and $f \cdot A_{430}$ at $140 \min \left(\left(f \cdot A_{430}\right)_{140 \min }\right)$ for the catechinone preparation in water/ethanol solution $\left(x_{\mathrm{A}}=0.28\right)$ with MEA. Here, the values of $f \cdot A_{430}$ at 140 min are adopted, because $f \cdot A_{430}$ increases and then decreases with reaction time at higher temperature and the downward turn begins before $200 \mathrm{~min}$ as described at the next section. The amount of the formed dye increases with increasing $T$ up to $30^{\circ} \mathrm{C}$ and it decreases with increasing $T$ over $30^{\circ} \mathrm{C}$. The formation of colourless products and/or the precipitation of colourants may be caused by the further reactions of $(+)$-catechin and catechinone, and such the side reactions give rise to the decrease in $f \cdot A_{430}$ at higher temperature [6]. In fact, the maximal value of $f \cdot A_{430}$ decreases with $T$. This indicates that higher temperature promotes the reactions too much. It can be said in conclusion that $30^{\circ} \mathrm{C}$ is the most preferable for producing the dyestuff in the water/alcohol mixed solution. 


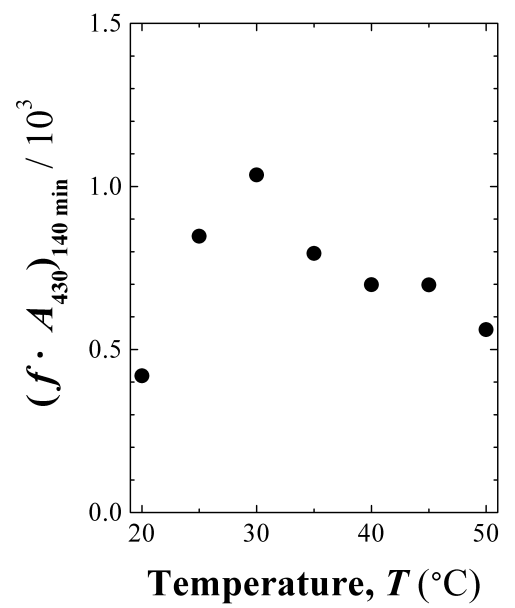

Figure 3. Relationship between reaction temperature $(T)$ and $\left(f \cdot A_{430}\right)_{140 \mathrm{~min}}$ for catechin preparation in water/ethanol mixed solution with MEA at $x_{\mathrm{A}}=0.28$.

\subsection{Effect of Alcohol Content and Sorts of Alcohols}

Figure 4 shows the time course of change in the amount of the formed dye $\left(f \cdot A_{430}\right)$ for catechinone preparation in water/ethanol mixed solution with MEA. The $x_{\mathrm{A}}$ was changed from 0.10 to 0.97 . The dye formation behaviour strongly depends on the $x_{\mathrm{A}}$ as shown in the figure. The $f \cdot A_{430}$ increases monotonously with $t$ at $x_{\mathrm{A}}=0.10$ 0.20 and $0.69-0.97$. On the other hand, $f \cdot A_{430}$ increases and then decreases with $t$ at $x_{\mathrm{A}}=0.25-0.59$. The maximal value of $f \cdot A_{430}$ also changes with $x_{\mathrm{A}}$ of $0.25-0.59$ and the maximum of $f \cdot A_{430}$ is given at $x_{\mathrm{A}}=0.25$. The formation of colourless products and/or the precipitation of colourants as described above may be caused also here leading to the decrease in $f \cdot A_{430}$ at $x_{\mathrm{A}}=0.25-0.59$.

Figure 5 depicts the relationship between $x_{\mathrm{A}}$ and $f \cdot A_{430}$ obtained at $140 \mathrm{~min}\left(\left(f \cdot A_{430}\right)_{140 \mathrm{~min}}\right)$ in water/ethanol mixed solution with MEA. The reason why the value of $f \cdot A_{430}$ obtained at $140 \mathrm{~min}$ is also adopted here, is that the same experiments were made in water and other alcohols as described below and the reactivities can be compared by using $\left(f \cdot A_{430}\right)_{140 \mathrm{~min}}$. The $\left(f \cdot A_{430}\right)_{140 \mathrm{~min}}$ increases steeply from $x_{\mathrm{A}}=0.10$ to 0.25 and decreases from 0.28 to 0.97 . The results show that the amount of formed dye depends strongly on the ethanol molar fraction and reaches its peak at $x_{\mathrm{A}}=0.25$. The results are interesting and it is thought that the effect of alcohol content for the reaction consists of two factors at least such as promotion and depression. Further, the same experiments were carried out using other alcohols being miscible with water such as methanol, 1-propanol, 2-propanol and tert-butyl alcohol. The results exhibit that the catechinone can be produced efficiently by adding all the alcohols used here and time change of $f \cdot A_{430}$ for the alcohols is in a similar manner as in the water/ethanol solution. The $f \cdot A_{430}$ for each reaction in the water/alcohol solutions increases monotonously with time, or reaches a peak and decreases. The $f \cdot A_{430}$ value for water/1-propanol solution at $x_{\mathrm{A}}=0.15$ takes a downward turn after $140 \mathrm{~min}$ as an earliest beginning of the decrease. Therefore, the value of $\left(f \cdot A_{430}\right)_{140 \mathrm{~min}}$ is adopted in order to make better comparison of the whole results of $f \cdot A_{430}$ under increasing. Figure 6 shows the relationships between the alcohol molar fraction for each of the mixed solvents $\left(x_{\mathrm{A}}\right)$ and $\left(f \cdot A_{430}\right)_{140 \mathrm{~min}}$ for each of the reactions made in each water/ alcohol mixed solution. The $\left(f \cdot A_{430}\right)_{140 \mathrm{~min}}$ for all the systems shows maximum against $x_{\mathrm{A}}$. The $\left(f \cdot A_{430}\right)_{140 \mathrm{~min}}$ represents the maximal value at $0.45,0.25,0.20,0.15$ or 0.10 of $x_{\mathrm{A}}$ for the $\mathrm{MeOH}, \mathrm{EtOH}, 2-\mathrm{PrOH}, 1-\mathrm{PrOH}$ or $t-$ $\mathrm{BuOH}$-mixed system, respectively. It is, then, important to clarify the basis for the order of the $x_{\mathrm{A}}$ values. The change in $\left(f \cdot A_{430}\right)_{140 \min }$ as shown in Figure 6 might relate with the concentration of $\mathrm{O}_{2}$ in the solution, the polarity of the mixed solvent, and/or a particular microstructure formed in the reaction solution. The authors study the mechanism and the reason for obtaining the results as shown in Figure 5 and Figure 6 at present.

Next, the dependence of the maximal amount of formed dye on the concentration of (+)-catechin in the reaction solution with the alcohols was investigated to elucidate the optimum condition for producing the dyestuff. $\mathrm{MeOH}, \mathrm{EtOH}$ and 1-PrOH are convenient linear alcohols and they were employed in the experiments as at each $x_{\mathrm{A}}=0.45,0.25$ or 0.15 , respectively for obtaining maximum. Figure 7 exhibits the relationships between the concentration of $(+)$-catechin (molarity: $\left.m_{S}\right)$ and the maximal amount of formed dye $\left(\left(f \cdot A_{430}\right)_{\max }\right)$ in the three 


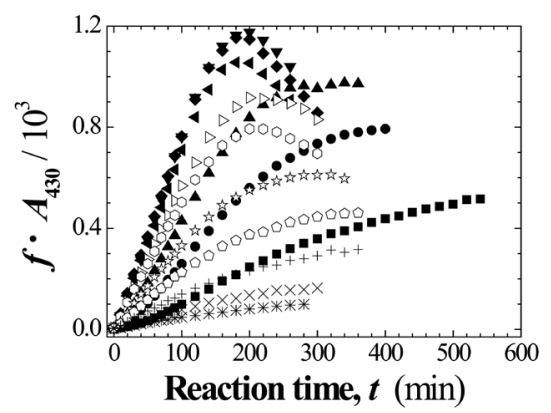

Figure 4. Reaction time $(t)$ change in the amount of the formed dye $\left(f \cdot A_{430}\right)$ in $(+)$-catechin and MEA water/ethanol mixed solution with $\mathrm{O}_{2}$ gas introduced at $30^{\circ} \mathrm{C}$. The ethanol molar fraction $\left(x_{\mathrm{A}}\right)$ of the mixed solvent is $0.10(\boldsymbol{\square}), 0.15$ ( $), 0.20(\boldsymbol{\Delta}), 0.25(\boldsymbol{\nabla}), 0.28(\diamond), 0.35(\boldsymbol{\Delta}), 0.40(\triangleright), 0.49$ (○), $0.59(\succsim), 0.69(\Delta), 0.79(+), 0.89(\times)$ or $0.97(*)$.

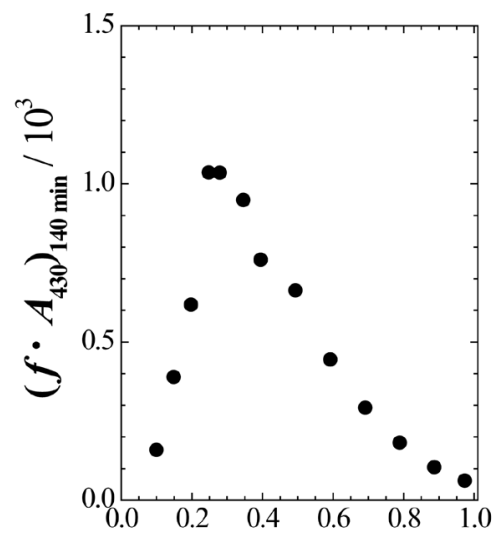

Alcohol molar fraction, $\boldsymbol{x}_{\mathrm{A}}$

Figure 5. Relationship between alcohol molar fraction $\left(x_{\mathrm{A}}\right)$ of the water/ethanol mixed solvent and the amount of the catechinone at $140 \mathrm{~min}\left(\left(f \cdot A_{430}\right)_{140 \mathrm{~min}}\right)$.

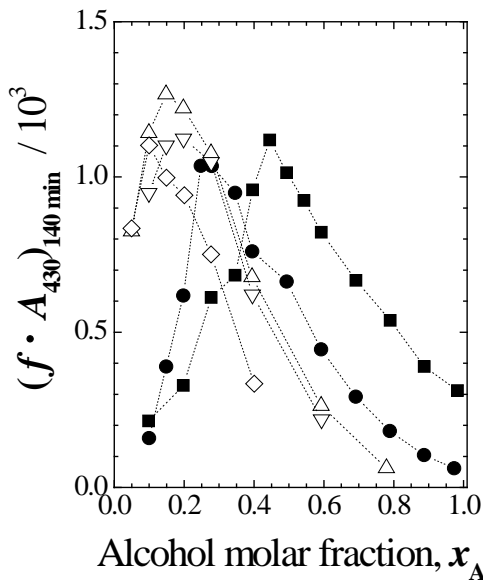

Figure 6. Relationship between $x_{\mathrm{A}}$ and $\left(f \cdot A_{430}\right)_{140 \mathrm{~min}}$ for the dye production at 140 min with $\mathrm{O}_{2}$ gas introduced at $30^{\circ} \mathrm{C}$ in the water/methanol ( $\square$ ), water/ethanol $(\mathbf{O})$, water/1-propanol $(\triangle)$, water/2-propanol $(\nabla)$ or water/tert-butyl alcohol $(\diamond)$ solution with MEA. 


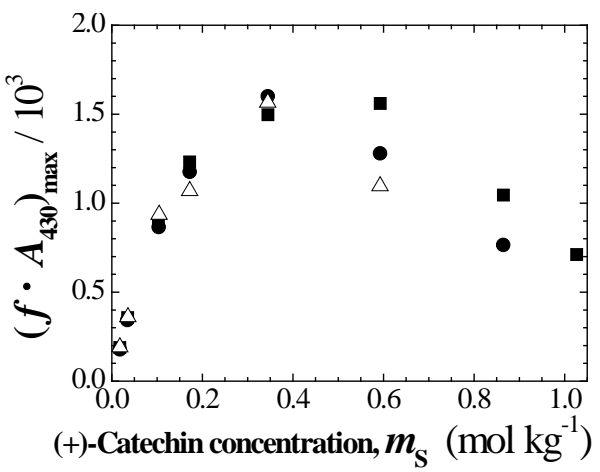

\begin{abstract}
Figure 7. Relationship between maximum of $f \cdot A_{430}\left(\left(f \cdot A_{430}\right)_{\max }\right)$ and the concentration of $(+)$-catechin $\left(m_{\mathrm{S}}\right)$ for the dye formation in water/alcohol mixed solution with MEA at $30^{\circ} \mathrm{C}$. The $x_{\mathrm{A}}$ is $0.45(\square), 0.25(-)$ or $0.15(\triangle)$ for the methanol, ethanol or 1-propanol added system, respectively.
\end{abstract}

kinds of water/alcohol systems. The maximum value of $f \cdot A_{430}$ is $c a .1600$ in all the reaction systems. The $m_{\mathrm{S}}$ value to give the maximum $f \cdot A_{430}$ for water/EtOH and water/1-PrOH systems is $0.35 \mathrm{~mol} \cdot \mathrm{kg}^{-1}$ and the values for water $/ \mathrm{MeOH}$ system are 0.35 and $0.59 \mathrm{~mol} \cdot \mathrm{kg}^{-1}$.

The results show that the amount of formed catechinone depends on the sort of alcohols, the alcohol molar fraction and the concentration of (+)-catechin, and there are optimum $x_{\mathrm{A}}$ and $m_{\mathrm{S}}$ values to obtain the dyestuff efficiently. It can be said that the EtOH is most preferable as the solvent added for the production of the dyestuff, because its safety is highest, its handling is easiest during and after the production and it is most economical.

\title{
4. Conclusion
}

The relationships between the amount of the formed catechinone and the dye preparation conditions such as basicity, sorts of added base, temperature, alcohol molar fraction of solvent and sorts of added alcohols were studied for the oxidation reaction of (+)-catechin in water/alcohol solution. The amount of catechinone obtained increases with increasing basicity and the dyestuff is obtained by adding MEA, DEA, TEA, L-arginine, $\mathrm{Na}_{2} \mathrm{CO}_{3}$, $\mathrm{K}_{2} \mathrm{CO}_{3}, \mathrm{Na}_{3} \mathrm{PO}_{4}$ or $\mathrm{NaOH}$. The optimum temperature for the production in water/ethanol solution is $30^{\circ} \mathrm{C}$. The optimum alcohol molar fraction of the mixed solvent for the dye formation is $0.45,0.25,0.20,0.15$ or 0.10 , with the methanol, ethanol, 2-propanol, 1-propanol or tert-butyl alcohol system, respectively. The amount of the obtained dyestuff reaches maximum at 1) 0.35 and 0.59 , 2) 0.35 or 3) $0.35 \mathrm{~mol} \cdot \mathrm{kg}^{-1}$ of the fed concentration of (+)-catechin for the 1) water/MeOH, 2) water/EtOH or 3) water/1-PrOH system, respectively.

\section{Acknowledgements}

This study was financially supported partly by the Japan Society for the Promotion of Science Research Foundation Grant (No. 21500732) and partly by Japan Science and Technology Agency as Adaptable \& Seamless Technology Transfer Program through Target-driven R \& D (No. AS2211611E). Japanese patent application number: 2012-219084 (12 November 2012).

\section{References}

[1] Corbett, J.F. (1971) Hair Dyes. In: Venkataraman, K., Ed., The Chemistry of Synthetic Dyes Vol. V, Academic Press, Inc., New York, 475-495. http://dx.doi.org/10.1016/B978-0-12-717005-3.50014-0

[2] Robbins, C.R. (2012) Chemical and Physical Behavior of Human Hair. 5th Edition, Springer-Verlag, Berlin Heidelberg, 447-464. http://dx.doi.org/10.1007/978-3-642-25611-0

[3] Søsted, H., Agner, T., Andersen, K.E. and Menné, T. (2002) 55 Cases of Allergic Reactions to Hair Dye: A Descriptive, Consumer Complaint-Based Study. Contact Dermatitis, 47, 299-303. http://dx.doi.org/10.1034/j.1600-0536.2002.470508.x

[4] Ishida, W., Makino, T. and Shimizu, T. (2011) Severe Hair Loss of the Scalp Due to a Hair Dye Containing Para Phenylenediamine. ISRN Dermatology, 2011, Article ID: 947284. http://dx.doi.org/10.5402/2011/947284 
[5] Yasunaga, H., Takahashi, A., Ito, K., Ueda, M. and Urakawa, H. (2012) Hair Dyeing by Using Catechinone Obtained from (+)-Catechin. Journal of Cosmetics, Dermatological Sciences and Applications, 2, 158-163. http://dx.doi.org/10.4236/jcdsa.2012.23031

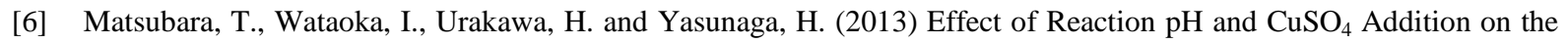
Formation of Catechinone Due to Oxidation of (+)-Catechin. International Journal of Cosmetic Science, 35, 362-367. http://dx.doi.org/10.1111/ics.12051

[7] Matsubara, T., Wataoka, I., Urakawa, H. and Yasunaga, H. (2014) High-Efficient Chemical Preparation of Catechinone Hair Dyestuff by Oxidation of (+)-Catechin in Water/Ethanol Mixed Solution. Sen'i Gakkaishi, 70, $19-22$. http://dx.doi.org/10.2115/fiber.70.19

[8] Perrin, D.D. and Dempsey, B. (1974) Buffers for pH and Metal Ion Control. Chapman \& Hall Ltd., London, 161-162.

[9] Mochizuki, M., Yamazaki, S., Kano, K. and Ikeda, T. (2002) Kinetic Analysis and Mechanistic Aspects of Autoxidation of Catechins. Biochim. Biophys. Acta-General Subjects, 1569, 35-44. http://dx.doi.org/10.1016/S0304-4165(01)00230-6 
Scientific Research Publishing (SCIRP) is one of the largest Open Access journal publishers. It is currently publishing more than 200 open access, online, peer-reviewed journals covering a wide range of academic disciplines. SCIRP serves the worldwide academic communities and contributes to the progress and application of science with its publication.

Other selected journals from SCIRP are listed as below. Submit your manuscript to us via either submit@scirp.org or Online Submission Portal.
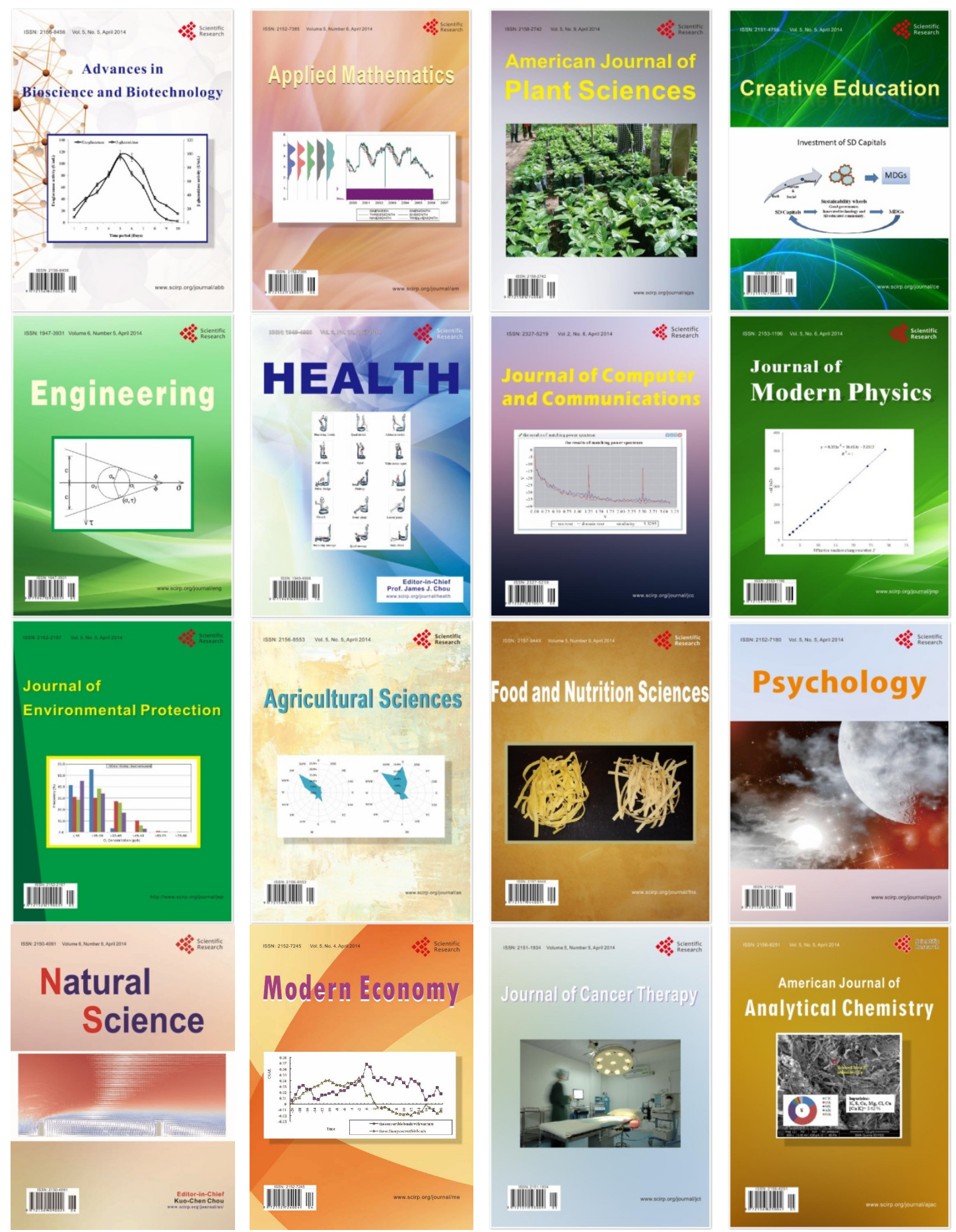